\title{
Après la sécheresse des années 1984-1987 dans le bassin de la Garonne (France)
}

\author{
Recommandations pour l'avenir
}

\author{
Henri Tardieu, Bertrand Plus \\ Compagnie d'aménagement des coteaux de Gascogne
}

\begin{abstract}
Des réservoirs insuffisamment remplis plusieurs années de suite, les besoins d'irrigation non satisfaits, le débit des grandes rivières et de la Garonne elle-même trop faible pour garantir l'alimentation en eau potable et la santé publique, des mesures d'interdiction des irrigations conduisant à des pertes économiques importantes, tels sont les faits majeurs de la période de sécheresse qui a commencé pendant l'été 1984 et s'est achevée en 1987. Ils ont frappé l'opinion publique et lui ont fait prendre conscience de l'importance des problèmes posés par l'insuffisance des ressources en eau.

L'aménageur se doit de bien mesurer le phénomène et de rechercher les meilleures solutions pour y répondre.
\end{abstract}

Le présent article comporte, en première partie, une courte analyse hydro-climatologique du phénomène. On verra que, même si l'événement paraît exceptionnel aux contemporains, l'analyse des longues séries statistiques montre qu'il doit être pris en compte car il est appelé à se reproduire en moyenne tous les vingt ans.

En deuxième partie, après l'interrogation concernant l'efficacité de la mesure d'urgence consistant à interdire les irrigations, nous indiquerons quelques éléments de la stratégie mise en place depuis cette période : outre les efforts nécessaires pour améliorer la gestion des eaux en temps réel, il paraît essentiel de mieux garantir la fiabilité des aménagements existants ou futurs.

\section{After the drought of the years 1984-1987 in the Garonne Basin. Recommendations for the future}

Reservoirs not sufficiently filled up several years running, irrigation needs left unsatisfied, the flow rate of the big rivers and the Garonne itself too weak to guarantee supplies of drinking water and public health, measures banning irrigation leading to big economic losses: these are the main features of the dry period which began in the Summer of 1984 and which ended in 1987.

In the first part of the present article we present a short hydro-climatological analysis of the phenomenon. We will see that it must be taken into account because it will occur 5 times every 100 years.

In the second part, after asking how efficient emergency measures which forbid irrigation are, we give some elements of the strategy set up since this period. 


\section{Quelques caractéristiques de la sécheresse 1984- 1987 dans le bassin de la Garonne}

Nous retiendrons, pour caractériser la récente période de sécheresse, les variables suivantes dont nous donnons les périodes de retour estimées après vérification de l'indépendance des valeurs annuelles successives (coefficient d'auto-corrélation de rang 1 inférieur à 0,1 ) :

- la pluviométrie annuelle sur l'année hydrologique ( $1^{\text {er }}$ octobre au 30 septembre):

- la pluviométrie estivale (juin, juillet, août) caractéristique des besoins en eau d'irrigation;

- l'écoulement moyen annuel des rivières caractéristique du niveau de remplissage des réservoirs réalisés en zone irriguée ;

- l'écoulement moyen pendant la période des irrigations (juin, juillet, août) caractéristique de la ressource en eau naturelle disponible, en provenance des Pyrénées, pour l'irrigation par prélèvement au fil de l'eau.

Le phénomène le plus rare est la sécheresse de l'été 1986 dont la période de retour est de l'ordre de 30 ans. Elle a entraîné des besoins en eau d'irrigation particulièrement élevés pendant l'été $1986\left(2070 \mathrm{~m}^{3} / \mathrm{ha}\right.$ pour une moyenne sur les 12 dernières années de $1280 \mathrm{~m}^{3} / \mathrm{ha}$ ). Les pluviométries annuelles sont proches de leur valeur décennale pendant trois années successives. Cela se traduit au niveau des écoulements par un phénomène de rémanence avec une augmentation progressive des périodes de retour.

Les réservoirs étant généralement calculés pour une garantie de remplissage de 4 années sur 5 , quelques-uns n'étaient pas pleins au début de l'été 1986. La plupart ont été insuffisamment remplis au début de l'été 1987, créant ainsi une nouvelle pénurie alors que les besoins en eau d'irrigation ont été proches de la moyenne.

Ces quelques caractéristiques montrent que, hors la sécheresse exceptionnelle de l'été 1986, chaque année prise isolément n'aurait pas dû conduire aux graves difficultés rencontrées au cours de cette période. C'est bien la succession de ces trois années qui crée un caractère de sécheresse exceptionnelle auquel l'aménagement actuel des ressources en eau n'a pas permis de faire face.

Pour élaborer une stratégie d'avenir face à un tel phénomène, inconnu depuis 1960, c'est-à-dire depuis le démarrage d'un développement significatif des irrigations, il faut bien sûr chercher à évaluer sa période de retour.
La brièveté des échantillons concernant la mesure des écoulement ne permet pas de faire cette analyse sur le débit des rivières (22 années de mesure).

En revanche, l'utilisation de longues séries pluviométriques (110 ans pour l'une d'entre elles) permet, en calculant la moyenne mobile sur 3 ans, d'établir le graphique I cicontre.

On note que la période de retour des 3 années (19841987) est voisine de 20 ans. Le graphique montre que, malheureusement, de telles successions se sont retrouvées cinq fois pendant le siècle passé et que si la sécheresse récente a tant frappé les esprits, c'est qu'elle a fait suite à une décennie (1974-1984) nettement plus humide que la moyenne.

On doit souligner ici que l'analyse d'un phénomène hydrologique récent au Maroc conduit à des conclusions assez semblables : sur le flanc nord du Haut Atlas, on doit relativiser l'impression d'exceptionnelle rareté qui a marqué la sécheresse des années 1981-1984 [9].

\section{Stratégie pour répondre à ce phénomène de séche- resse exceptionnel?}

Fort d'analyses statistiques montrant le caractère exceptionnel de la sécheresse, on pourrait considérer qu'il s'agit d'une " catastrophe naturelle " justifiant l'appel à la solidarité nationale et ne nécessitant pas de réponse en terme d'aménagement.

Autrement dit, les ingénieurs se préoccuperaient de faire face aux sécheresses de fréquence de retour supérieure à une année sur cinq, laissant à d'autres la résolution des problèmes posés par les sécheresses exceptionnelles. Cette réponse ne résiste pas à l'analyse car, en cas de sécheresse grave, toutes les activités consommatrices d'eau sont en conflit. Il faut donc avoir en main une méthode pour répondre aux urgences, afficher des priorités, être capables de les tenir.

Par ailleurs, le soutien économique apporté par les caisses de "calamité agricole " ne concernera pas la culture insuffisamment irriguée, car ce sont les cultures non irriguées, encore plus sinistrées, qui requièrent l'aide de l'Etat. Cette situation rend paradoxalement les irrigants plus vulnérables aux sécheresses exceptionnelles.

\begin{tabular}{|l|c|c|c|r|}
\hline \multicolumn{3}{|c|}{ Période de retour en années } \\
\hline \multirow{2}{*}{ Années } & \multicolumn{2}{|c|}{ Pluviométrie } & \multicolumn{2}{c|}{ Ecoulements } \\
& annuelle & Mois $6 / 7 / 8$ & annuels & mois $6 / 7 / 8$ \\
\cline { 2 - 5 } $1984-1985$ & 8 & 5 & 3 & 4 \\
$1985-1986$ & 9 & 30 & 6 & 15 \\
$1986-1987$ & 7 & 2 & 17 & n.c. \\
\cline { 2 - 5 } & & & & \\
\end{tabular}




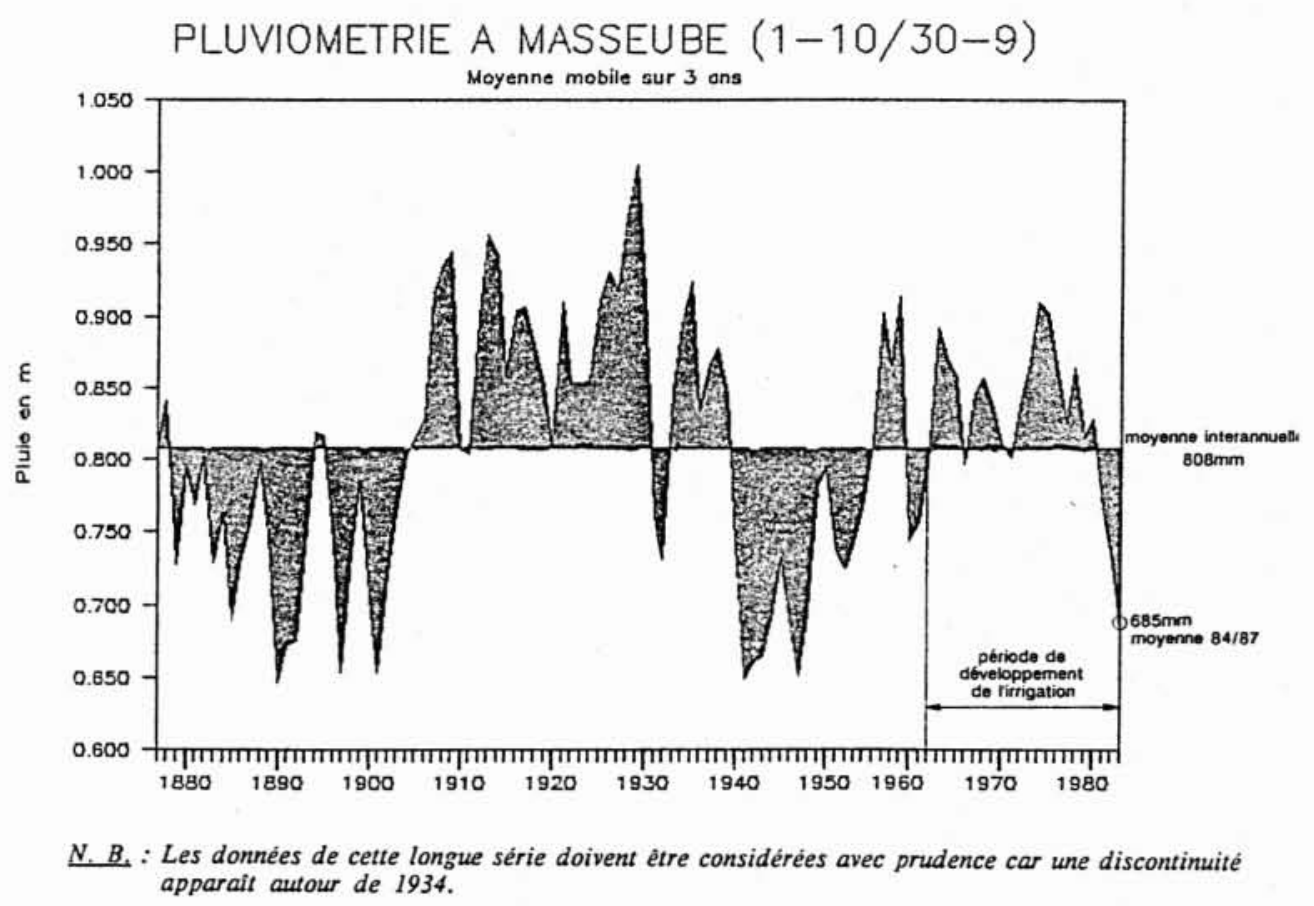

1.

Il est donc indispensable de préparer une stratégie de réponse aux sécheresses exceptionnelles visant à définir les priorités dans les usages de l'eau pour sauvegarder la santé publique, et à minimiser les pertes économiques sur l'agriculture.

Cette stratégie concerne d'une part les interventions d'urgence, d'autre part l'adaptation des stratégies d'aménagement.

\subsection{Une réponse d'urgence : l'interdiction des irrigations?}

Les rivières du bassin Garonne ont toutes une vocation mixte de transport d'eau pour l'irrigation et de milieu récepteur pour les prélèvements et rejets d'eau publics (AEP et industrie) [3].

Aussi, devant les graves inquiétudes provoquées par la sécheresse 1984-1987 pour l'alimentation en eau potable, l'autorité administrative a été obligée d'interdire les irrigations bien avant la fin de la campagne.

L'enjeu économique d'une telle décision est énorme. Pour la région concernée, le produit brut des cultures irriguées est voisin de $800 \mathrm{MF} / \mathrm{an}$. Si la décision administrative conduit à une perte de récolte de $50 \%$, on comprend qu'elle ne soit pas applicable facilement et conduise à des "résistances" sur le terrain. Ce fut le cas en 1986, où de très fortes tensions sont apparues.

Pour mieux résoudre les conflits, l'expérience de ces années de sécheresse nous conduit à faire les recommandations suivantes :
- se donner les moyens techniques de suivre en temps réel l'évolution des phénomènes climatiques, l'évolution des réserves en eau et des débits des cours d'eau, le niveau réel des prélèvements pour l'irrigation et l'eau potable. Il s'agit de faire de la "gestion des eaux en temps réel " afin de pouvoir informer les différents acteurs du caractère exceptionnel de la situation ;

- avoir défini auparavant des stratégies de réponse précise permettant de prendre les décisions au plus près des intérêts économiques en jeu : une semaine gagnée en août avant d'interdire les irrigations peut conduire à un accroissement de risque négligeable en regard d'un avantage économique très significatif ;

- provoquer la concertation de tous les acteurs pour éviter des comportements anarchiques conduisant à des pertes d'eau et pour permettre la répartition équitable des ressources disponibles (exemples: irrigation excessive quand les agriculteurs "sentent" qu'une décision d'interdiction va intervenir, manauvres intempestives des vannes par les usiniers au fil de l'eau nuisant à la bonne régulation des débits des rivières).

L'observation de ces trois règles, mises progressivement en pratique au cours de cette période 1984-1987, conditionne le succès d'une décision de restriction rendue nécessaire pour préserver la santé publique. 


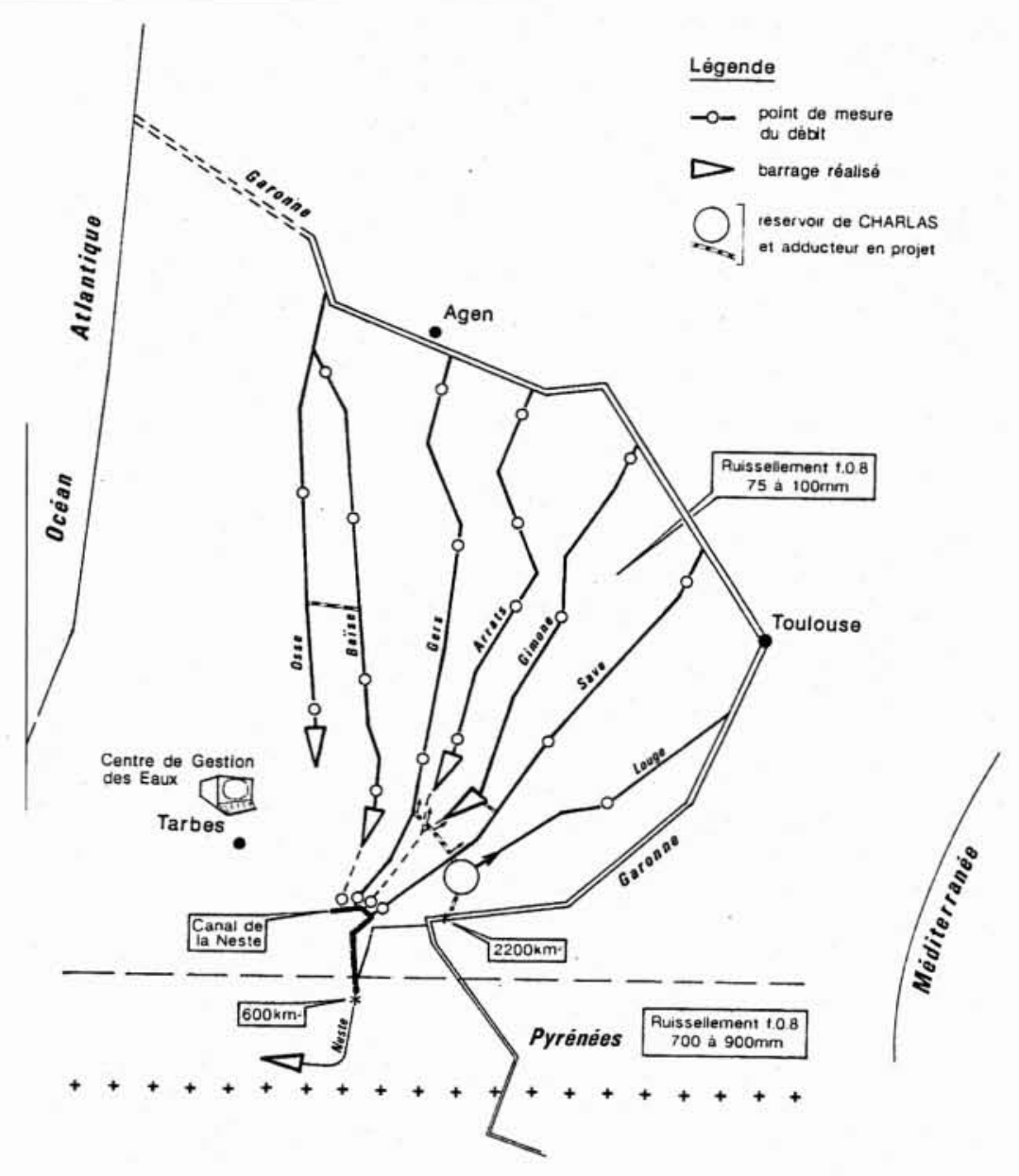

2. Gestion des eaux Neste-Garonne.

\subsection{Les mesures préventives pour répondre aux sécheresses exceptionnelles}

La réponse simple de l'aménageur pourrait être « stockons plus d'eau ". Les contraintes économiques conduisent à envisager en préalable les mesures d'amélioration de la gestion des eaux, indispensables pour démontrer que la création de nouveaux stockages n'est que l'achèvement d'une démarche complète d'économie d'eau:

\subsubsection{Améliorer la gestion de l'eau}

a) Former les agriculteurs à gérer un assolement sous contrainte de débit et de volume d'eau maximum: Dans l'optimisation des assolements, l'eau est souvent considérée comme un intrant «banal " à prix défini et à disponibilité illimitée comme les engrais. La consommation optimum est alors celle dont le coût annule la productivité marginale. Or, en cas de sécheresse requérant une limitation de la consommation d'eau, on est conduit, faute de pouvoir agir efficacement sur le prix, à considérer l'eau disponible en débit et volume comme une contrainte physique au même titre que la surface cultivable.

Depuis 1986, la CACG - en collaboration avec les instituts de recherche - développe ce thème de réflexion et entreprend des programmes de formation [8]. D'ores et déjà, la notion de "quota d'eau disponible " passe dans les esprits ; le quota d'eau devient aussi important, ou parfois plus important, que la surface exploitable disponible. Ceci conduit à rechercher des assolements plus économes en eau valorisant au mieux les périodes pluvieuses et les réserves du sol, mais surtout à mieux diffuser auprès des irrigants, le raisonnement économique de valorisation de l'eau : on peut espérer une plus grande souplesse d'adaptation des assolements et une meilleure conduite des irrigations dans les cas de manque d'eau prévisibles (celles des "sécheresses" qui sont provoquées par un remplissage insuffisant des réservoirs prévisible dès le mois de mars). 
b) Poursuivre le développement des systèmes de gestion automatique des ouvrages: Depuis 1984, la CACG développe les systèmes de gestion automatique des réservoirs en temps réel. L'expérience montre que cela conduit ả un gain de l'ordre de $20 \%$ sur l'utilisation des volumes stockés. II s'agit bien, par une meilleure gestion des réservoirs, de "créer" une ressource nouvelle provenant d'une moindre perte d'eau à l'aval. L'eau récupérée provient notamment d'une anticipation sur les besoins d'irrigation et d'une meilleure utilisation des débits naturels disponibles pendant la période d'irrigation [1], [5].

c) Développer les outils d'aide à la décision concernant les stratégies optimum d'utilisation des ressources en eau: Analyser la situation hydrologique et climatique, esquisser des prévisions de consommation et de ressource, apprécier les risques futurs et leurs enjeux économiques, arbitrer l'ensemble pour définir une stratégie optimum de réponse à la sécheresse, tout cela ne s'improvise pas. L'expérience récente nous l'a montré à propos de la gestion complexe du système Neste qui comporte une dizaine d'ouvrages de stockage de capacité totale voisine de 80 millions de mètres cubes et des ouvrages de transfert dont un canal de $14 \mathrm{~m}^{3} / \mathrm{s}$ équipé d'une quinzaine d'organes de restitution d'eau.

La mise au point, en cours, d'un modèle d'optimisation des stratégies en temps réel, devrait conduire à prendre de meilleures décisions d'utilisation des ouvrages et de restrictions éventuelles des usages. Ce modèle, travaillant au pas de temps de la semaine à l'horizon de quelques mois, est fondé sur une double approche :

- définition de "bonnes stratégies" fondées sur une analyse du passé permettant de situer en fréquence la stratégie actuelle avec quantification de l'éventail des possibles. La réduction des incertitudes est accrue par les quelques modèles de prévisions possibles (prévision des étiages, des consommations d'eau d'irrigation).

- optimisation d'une fonction économique permettant, dans le lot des " bonnes " stratégies, de retenir la meilleure par les méthodes de programmation dynamique [2], [4], [7].

\subsubsection{Mieux penser les programmes d'aménagement}

Au-delà des mesures visant à l'amélioration de la gestion des eaux, la période récente a conduit - bien normalement - à accélérer le programme de création de barrages nouveaux permettant de faire face à l'accroissement des besoins. Mais la sécheresse a également mis en lumière la nécessité de mieux garantir la fiabilité des ouvrages existants.

a) Assurer la maintenance des ouvrages anciens : Le canal de la Neste (réalisé en 1860) prélève 250 millions de mètres cubes sur les eaux descendant des Pyrénées pour les distribuer sur les affluents rive gauche de la Garonne, desservant ainsi une zone de près de un million d'hectares. Sur ce volume, moins de 50 millions de mètres cubes proviennent de stockage. Il s'agit donc d'un ouvrage de transfert qui valorise considérablement les eaux naturelles. Alors que toutes les énergies sont mobilisées pour créer des stockages nouveaux, au rythme de 10 à 15 millions de $\mathrm{m}^{3}$ tous les 5 ans afin d'assurer le développement des irrigations, la maintenance du Canal de la Neste était quasiment abandonnée.

Perçu comme appartenant au paysage «naturel » de la Gascogne, il a été négligé au profit des ouvrages nouveaux. La sécheresse récente a rappelé que les énergies dépensées pour poursuivre la réalisation du programme de barrage sont vaines si le canal de la Neste ne remplit plus son office de répartition du débit naturel.

Un programme décennal de restauration et de modernisation a été mis en place en 1988 en vue de sauvegarder cet ouvrage. Son coût de 250 millions de francs, rapporté au mètre cube de débit naturel valorisé soit $1,20 \mathrm{~F} / \mathrm{m}^{3}$, est faible par rapport au coût d'un stockage de substitution $\left(=7 \mathrm{~F} / \mathrm{m}^{3}\right)[6]$.

b) Viser une meilleure garantie de remplissage des réservoirs: Les mesures de limitation ou d'interdiction des irrigations peuvent être dues à une pluviométrie estivale exceptionnellement faible, ou plus précisément inférieure à celle qui a été prise en compte pour calculer les besoins en eau théoriques maximum auxquels doit faire face l'aménagement (en général, pluviométrie non dépassée l année sur 5). Elles peuvent aussi être dues à un mauvais remplissage des réservoirs; elles sont alors très difficiles à "faire passer " auprès des irrigants car elles mettent en cause la crédibilité même des aménagements.

Dans l'établissement des schémas d'aménagement, il convient done de prendre en considération une très bonne garantie de remplissage des réservoirs (au moins 9 années sur 10) pour laisser comme seul risque important celui qui provient d'un besoin en eau supérieur à celui qui a été prévu.

Pour les réservoirs existants remplis par leur bassin versant propre, il a été réalisé, depuis la sécheresse, des systèmes peu coûteux de transfert des eaux naturelles provenant des grands bassins versants voisins.

Par une optimisation fine des volumes d'eau disponibles dans la rivière, des coûts de pompage suivant les périodes tarifaires de l'électricité et d'une gestion interannuelle des volumes stockés, on peut atteindre une garantie de remplissage très supérieure à $9 / 10$ moyennant un coût de pompage complémentaire en cas d'hiver sec. Ce coût est faible par rapport à la perte de revenu évitée.

Pour l'aménagement d'ensemble de la région des Coteaux de Gascogne, ce principe conduit à rechercher, pour le remplissage des futurs réservoirs, un apport d'eau extérieur à la région. En effet, sauf utilisation très locale, les bassins versants des zones irriguées sont devenus globalement insuffisants pour assurer la ressource en eau nécessaire (lame d'eau ruisselée garantie 4 années sur 5 $(f=0,8): 75 \dot{a} 100 \mathrm{~mm})$. Jusqu'ici, le complément a été apporté par le canal de la Neste qui draine vers les zones irriguées un bassin versant pyrénéen à forte hydraulicité (la Neste : $606 \mathrm{~km}^{2}$, lame d'eau ruisselée $\left.(f=0,8): 850 \mathrm{~mm}\right)$. Son maintien en état est d'autant plus indispensable qu'il assure aussi le remplissage des grands réservoirs de piémont (Astarac, Puydarrieux, Lunax). 
Pour l'avenir, la poursuite du développement des irrigations et l'amélioration de la fiabilité des aménagements existants exigent la mobilisation des eaux hivernales d'un bassin versant pyrénéen beaucoup plus grand, celui de la Garonne elle-même $\left(2200 \mathrm{~km}^{2}\right)$, lame d'eau ruisselée : $670 \mathrm{~mm}(f=0,8)$.

C'est le projet d'aménagement dit de Charlas comportant :

— un nouveau réservoir de 100 millions de mètres cubes, destiné pour moitié à la Gascogne et pour moitié à la Garonne ;
- un adducteur provenant de la Garonne, à Montréjeau, permettant de garantir le remplissage pendant la période de hautes eaux de la Garonne ;

- et un réseau de galeries distribuant l'eau suivant les besoins de chaque rivière et l'état des stocks répartis.

Outre le volume d'eau supplémentaire qu'il assure au Système Neste, ce nouvel ouvrage permet d'améliorer la fiabilité de l'ensemble des aménagements existants [3]. Sa fonction d'ouvrage de réalimentation de la Garonne, en amont de Toulouse, constitue le premier pas vers un soutien d'étiage de la Garonne, devenu indispensable.

\section{Conclusion}

La nécessité d'élaborer une stratégie de réponse aux sécheresses exceptionnelles ressort de quelques constats simples.

La période de sécheresse 1984-1987 a certes été grave, mais l'érosion de la mémoire collective a tendance à nous faire exagérer la rareté des phénomènes exceptionnels. Par ailleurs, les actions qui visent à nous protéger des excès de la nature nous y rendent paradoxalement plus vulnérables. Aussi, une défaillance des aménagements (insuffisance de remplissage des réservoirs) est beaucoup plus mal vécue par les utilisateurs que l'effet direct d'un manque de pluie pendant l'été : dès qu'il y a aménagement, on n'accepte plus la fatalité, et on recherche un bouc-émissaire, même s'il s'agit d'un risque naturel.

Or, la résolution des conflits nés de la sécheresse nécessite la confiance des irrigants dans les aménagements et en ceux qui les ont conçus. Car, s'il faut aux dirigeants de la fermeté pour prendre l'ultime mesure de sauvegarde (interdiction des irrigations pour préserver l'alimentation en eau potable), cette confiance des irrigants est indispensable pour la rendre efficace.
Ainsi, outre les efforts nécessaires pour améliorer la gestion des eaux en temps réel, il paraît essentiel de mieux garantir la fiabilité des aménagements existants ou futurs.

L'amélioration de la gestion des eaux vise notamment une meilleure valorisation des eaux naturelles :

- le volume d'eau d'irrigation garanti, ajouté à la pluie et à la réserve du sol, doit être valorisé par une optimisation des assolements sous contrainte d'apport d'eau limité ;

- la gestion automatique des réservoirs et la définition de stratégies optimales d'utilisation des ressources permettent de valoriser conjointement les débits naturels disponibles et les stockages d'eau; ces méthodes apportent une réponse en temps réel à la pénurie d'eau.

Une meilleure fiabilité des aménagements est recherchée :

- en assurant la maintenance, trop souvent négligée, des ouvrages anciens dont on a parfois tendance à oublier le rôle indispensable ;

- en améliorant la garantie de remplissage des réservoirs par une utilisation du débit naturel des rivières en période de hautes eaux et par des transferts locaux ou régionaux adaptés.

\section{Références bibliographiques}

[1] Piquereau A., Tardieu H., Villocel A. \& Verdier J. (1983). — « Réduction des pertes en eau par l'automatisation de la gestion d'un barrage réalimentant une rivière en vue de l'irrigation. Paper presented at the Twelfth Congress of the International Commission on Irrigation and Drainage FortCollins, Colorado (USA).

[2] Roche P.A., Masson J.M., Vermersch J.P. (1985). "Outils d'aide à la programmation des barrages réservoirs du bassin de la Seine ".

[3] C.A.C.G. (1987). - «Mobilisation des ressources en eau. Bilan et perspective après la sécheresse ", Revue Coteaux de Gascogne, $\mathrm{n}^{\circ} 73$.

[4] Parent E. (1987-1988). - "Procédures d'optimisation des consignes de gestion d'un barrage-réservoir ". Thèse en cours, CERGRENE.
[5] TARDieu H. (1988). - " Automatic dam management and river regulation for irrigation purposes ", Irrigation and Drainage Systems 2: 53-61, Martinus Nijhoff Publishers, Dordrecht (Netherlands).

[6] Bourla F.R. (1988). - " Le Canal de la Neste ", revue AdourGaronne, $\mathrm{n}^{\circ} 38$.

[7] Turgeon A., MaArouf SAad, (Hydro-Québec) (1988). "Application of principal component analysis to long term reservoir management ", Water Resources Research, volume $24 \mathrm{~N}^{\circ} 7$, pages 907-912.

[8] I.N.R.A., I.T.C.F., A.G.P.M., C.E.T.I.O.M., C.A.C.G. (19871988). - "Protocole d'expérimentation sur la gestion de l'eau " et modèles d'optimisation des assolements, rapports annuels.

[9] Motor Colombus, Coyne et Bellier, Ingema, C.I.D., juin 1988. - " Etude du Plan directeur intégré d'aménagements des eaux des bassins Sebou, Bou Regreg, Cum Erbia et Tensift, hydrologie du Tensift. 